\section{RMD Open}

Rheumatic \&

Musculoskeletal Diseases

\title{
Changes in bone mineral density over 10 years in patients with early rheumatoid arthritis
}

Lisa Theander (D) , ${ }^{1}$ Minna Willim, ${ }^{2,3}$ Jan Åke Nilsson, ${ }^{1,2}$ Magnus Karlsson, ${ }^{4,5}$ Kristina E Åkesson, ${ }^{4,5}$ Lennart T H Jacobsson, , ${ }^{1,6}$ Carl Turesson ${ }^{1,2}$

To cite: Theander L, Willim M, Nilsson JÅ, et al. Changes in bone mineral density over 10 years in patients with early rheumatoid arthritis. RMD Open 2020;6:e001142. doi:10.1136/ rmdopen-2019-001142

- Additional material is published online only. To view please visit the journal online (http://dx.doi.org/10.1136/

Received 4 November 2019 Revised 19 January 2020 Accepted 20 January 2020 rmdopen-2019-001142).

\section{ABSTRACT}

Objectives To investigate changes in bone mineral density (BMD) in patients with early rheumatoid arthritis (RA) over a 10-year period.

Methods Consecutive patients with early RA (symptom duration $<12$ months) were followed according to a structured programme and examined with dual-energy $X$-ray absorptiometry (DXA) at inclusion and after 2, 5 and 10 years. Mean Z-scores over the study period were estimated using mixed linear effect models. Changes in Z-scores between follow-up visits were analysed using paired T-tests.

Results At inclusion, 220 patients were examined with DXA. At the femoral neck, the mean Z-score over 10 years was $-0.33(95 \% \mathrm{Cl}-0.57$ to -0.08$)$ in men and -0.07 $(-0.22$ to 0.08$)$ in women. Men had significantly lower $\mathrm{BMD}$ at the femoral neck than expected by age at inclusion (intercept Z-score value $-0.35 ; 95 \% \mathrm{Cl}-0.61$ to -0.09 ), whereas there was no such difference in women. At the lumbar spine, the mean Z-score over the study period for men was -0.05 ( -0.29 to 0.19$)$ and for women 0.06 $(-0.10$ to 0.21$)$. In paired comparisons of BMD at different follow-up visits, femoral neck Z-scores for men decreased significantly from inclusion to the 5 -year follow-up. After 5 years, no further reduction was seen.

Conclusions In this observational study of a limited sample, men with early RA had reduced femoral neck BMD at diagnosis, with a further significant but marginal decline during the first 5 years. Lumbar spine BMD Z-scores were not reduced in men or women with early RA. Data on 10year follow-up were limited.

\section{INTRODUCTION}

Check for updates

\section{(c) Author(s) (or their} employer(s)) 2020. Re-use permitted under CC BY-NC. No commercial re-use. See rights and permissions. Published by BMJ.

For numbered affiliations see end of article.

\section{Correspondence to} Dr Lisa Theander; lisatheander@hotmail.com
Patients with rheumatoid arthritis (RA) have been shown to have an increased risk of osteoporosis and fractures. ${ }^{1-4}$ Although long standing and active, especially erosive, disease seems to be particularly predictive of osteoporosis in patients with $\mathrm{RA}^{3-6}$ higher loss of bone mass than expected by age has also been apparent soon after diagnosis in several cohorts. ${ }^{78}$ In a study of bone mineral density (BMD) over the first 10 years in patients diagnosed with RA in the 1980s and early 1990 s,

\section{Key messages}

What is already known about this subject?

- Patients with rheumatoid arthritis (RA) have been shown to have an increased risk of osteoporosis and fractures.

- Higher loss of bone mass than expected by age has been apparent soon after diagnosis in several cohorts.

What does this study add?

- In this study of patients diagnosed with RA after 1995, bone mineral density in the femoral neck was reduced at diagnosis of RA in men but not in women.

How might this impact on clinical practice?

- In men with RA, potential benefits of early intervention against bone loss should be further studied.

Kroot et al found that bone loss was most marked during the first 2years. ${ }^{9}$ A similar pattern was seen in a study conducted 10 years later (inclusion 1999-2001), where the annual rate of bone loss was higher during the first 2 years compared with the following 8 years. ${ }^{10}$ More aggressive antirheumatic treatment during the later part of the study period was suggested to contribute to this pattern. ${ }^{10}$ With the rapid progress in the management of patients with RA, including more and better options for treatment to remission, ${ }^{11}$ there is a persisting need for re-evaluation of the changes in BMD following RA diagnosis.

Osteoporosis affects both men and women, but there are important differences in incidence and in the course of bone loss. Women start losing bone at an earlier age and at a faster rate than men. ${ }^{12}$ Among men, factors associated with secondary osteoporosis, such as alcoholism, excessive smoking and various comorbidities, are more common than in women. ${ }^{13}$ Accordingly, there is a rational for separate analyses of BMD in men and women. 
BMD varies with age and sex. Z-scores (number of SD above or below the mean BMD for the given age and sex) enable comparisons of BMD from time to time and between different individuals, whereas T-scores give information on whether a patient suffers from osteoporosis or not according to the WHO definition. ${ }^{14}$ In previous studies, one SD decrease in BMD has been associated with roughly doubled fracture risk. ${ }^{1516}$

In this study, we have followed patients with recently diagnosed RA, treated according to the general recommendations, for 10 years with repeated BMD measurements (dual-energy X-ray absorptiometry (DXA)). The aim was to examine changes in BMD by sex over the first 10 years and to investigate whether patients with RA have lower BMD than expected already at diagnosis, whether BMD changes during the course of disease and which baseline factors predict changes in bone mass. Insights on these issues are of importance for further improvement of the management of bone health in patients with RA.

\section{MATERIALS AND METHODS Patients}

An inception cohort of consecutive patients with early RA ( $\mathrm{n}=233$, symptom duration $<12$ months), recruited between 1995 and 2005, was investigated. The catchment area was the city of Malmö, Sweden (population 260000 in the year 2000). Patients were recruited from the rheumatology outpatient clinic of Malmö University Hospital, the only hospital serving the city, or from the four rheumatologists in private practice in the area. All included patients were diagnosed by a rheumatologist and fulfilled the 1987 American College of Rheumatology criteria for RA. ${ }^{17}$ All patients were managed according to standard care without any prespecified protocol for antirheumatic treatment. The patients were included before the current practice of treat to target was implemented, ${ }^{18}$ and before early treatment with biological disease modifying antirheumatic drugs (bDMARDs) came into widespread use. Results on other outcomes in this cohort have been reported previously. ${ }^{19} 20$

\section{Clinical assessment}

The patients were examined at inclusion and after 6 , 12, 24, 60 and 120 months by the same rheumatologist according to a structured protocol. Disability was assessed using the Swedish validated version of the Health Assessment Questionnaire. ${ }^{21}$ Visual Analogue Scales were used to evaluate the patients' assessment of current pain and the patients' global assessment of disease activity. Information on height, weight, smoking history (ever/ never) and menopausal status was collected at inclusion through a self-administered questionnaire. Information on current use of synthetic disease modifying antirheumatic drugs (sDMARDs), glucocorticosteroids, antiosteoporotic agents and hormone replacement therapy (HRT) was obtained through a structured interview at each visit.
Information on use of bDMARDs during the study period was obtained from the South Swedish Arthritis Treatment Group register $^{22}$ and the Swedish Rheumatology Quality register. ${ }^{23}$

\section{Laboratory investigations}

Rheumatoid factor (RF) and antibodies to cyclic citrullinated peptides (anti-CCP) were analysed at inclusion using standard ELISA methods at the Immunology laboratories at the University Hospitals in Malmö and Lund. IgM RF was analysed using ELISA, which was calibrated against the WHO RF reference preparation. Anti-CCP antibodies were analysed using the Quanta Lite CCP IgG ELISA (INOVA Diagnostics, USA). Erythrocyte sedimentation rate and $\mathrm{C}$ reactive protein were assessed according to standard methods at the Department of Clinical Chemistry, Malmö University Hospital.

\section{Radiographic assessment}

Radiographic evaluation of hands and feet was carried out at inclusion and after 2, 5 and 10 years of follow-up. The presence of erosions (present versus absent) was determined by a radiologist, unaware of the clinical status of the patient, as part of standard clinical practice.

\section{Bone mineral density measurements}

At inclusion and after 2, 5 and 10 years, the patients were examined with DXA at the left femoral neck and second to fourth lumbar spine vertebrae (L2-L4). The majority of patients were measured by the same DXA equipment (Lunar DPX-L equipment, 1.3z Lunar, Madison, Wisconsin, USA) during the study period. The centre applies quality control by daily checking the stability of the systems using a manufacturer-supplied phantom. In accordance with recommendations, precision is assessed as previously described: CV\% $0.50 \%$ (total hip) and $0.65 \%$ (lumbar spine),${ }^{24}$ while higher in the very elderly. ${ }^{25}$ For practical reasons, 67 of the patients had all their measurements done on either the Lunar $D P X-N T$ equipment or Lunar Prodigy equipment. Seven of these patients had one measurement done on the Lunar Prodigy equipment and their other measurements on the Lunar DPX-NT equipment. Our analyses indicate that the difference between the machines is marginal (unpublished results). From the BMD values $\left(\mathrm{g} / \mathrm{cm}^{2}\right)$ Z-scores (number of SD above or below the mean BMD for the given age and sex) were calculated using a cohort of healthy individuals (146 men and 178 women, age 20-87) from the same area as a reference population. ${ }^{26}$ Gender-specific reference values were estimated using piecewise linear regression separately for patients aged 20-44 and $\geq 45$ in the femoral neck in men and women and in the lumbar spine in men, but for patients aged $20-44,45-64$ and $\geq 65$ in the lumbar spine in women. ${ }^{26}$ Outliers were managed according to standard procedures used in other studies. ${ }^{27}$ BMD values exceeding \pm 3 SD from the mean for the given age and sex were considered outliers and excluded from the analyses. Over the 
study period, three measurements in the femoral neck and three measurements in the lumbar spine were excluded for this reason.

\section{Statistics}

The mean Z-scores over the study period were estimated using mixed linear effect models, where the intercept corresponded to the estimated mean Z-score at baseline, based on the regression line. The impact of baseline characteristics on the mean Z-score over 10 years was analysed in univariate models. In order to assess potentially independent effects on BMD Z-scores over time, significant predictors in the univariate models were further evaluated in multivariate analyses. In addition, the two established risk factors for osteoporosis, smoking and (in women) postmenopausal status, were included in all multivariate models. To assess collinearity, correlations between parameters were analysed using Spearman's test.

To evaluate changes in BMD during specific phases of early RA ( $0-2$ years, $2-5$ years, $5-10$ years, $0-5$ years and 0-10 years), changes in Z-scores between follow-up visits for patients with data at both time points were analysed using the paired T-test. Analyses were performed for the femoral neck and L2-L4 separately and stratified by sex. Data are presented as mean $(95 \% \mathrm{CI})$. The analyses were performed using IBM SPSS statistics V.24.

\section{Ethics}

All patients gave their written informed consent to participate, and the study was approved by the Regional Ethical Review Board for southern Sweden (Lund, Sweden, LU 410-94, LU 311-02). The study was conducted according to the principles of the Helsinki Declaration.

\section{RESULTS}

Patients-baseline characteristics

A total of 233 patients were included in the cohort. Of these, 220 patients were examined with DXA at inclusion. Ten patients underwent the first DXA scan later during the study period, whereas three patients never came for any DXA examination. In three cases, BMD of the hip was not measured at inclusion. For men and women with baseline DXA measurements, the mean age at inclusion was 63.2 (SD 11.1) and 58.5 (SD 15.6) years, respectively, with a mean duration of symptoms of 7.1 (SD 2.8) and 7.5 (SD 2.9) months, respectively. Seventy-one per cent were women, whereof $73 \%$ (113/154) were postmenopausal. Baseline characteristics for patients with DXA data at each time point are shown in table 1 . Of the included men, $44.9 \%$ had been examined with DXA at all occasions whereas the corresponding number was $50.6 \%$ for women. A total of 50 patients (19 men and 31 women) died during the 10-year follow-up. Except for a lower age at inclusion, characteristics of those with DXA data at all evaluations were not substantially different from those with DXA data at inclusion (table 1).

\section{Treatment}

sDMARDs were used in over $80 \%$ of men and women at baseline. Forty-nine per cent of the men and $36 \%$ of the women were treated with glucocorticoids at baseline (table 1). The average daily dose Prednisone at baseline among men was $11.1 \mathrm{mg}$ and among women $8.0 \mathrm{mg}$. Treatment at every point of follow-up is presented in table 2 . Throughout the study period, 11 men and 37 women had been treated with biological DMARDs at some point and all of these except for one woman had been treated with at least one TNF-inhibitor. At the 5-year follow-up, 16.7\% of the men were treated with bisphosphonates, compared with $35.5 \%$ of the women.

\section{BMD over time}

Observed BMD Z-scores were numerically lower in men than in women at inclusion and at every point of follow-up (table 2). At the femoral neck, the mean Z-score over 10 years of time was $-0.33(-0.57$ to -0.08$)$ in men and -0.07 $(-0.22$ to 0.08$)$ in women (table 3$)$. Men had significantly lower BMD at the femoral neck than expected based on age at inclusion (estimated by the intercept Z-score value $-0.35,95 \% \mathrm{CI}-0.61$ to -0.09 ), whereas there was no significant overall change in femoral neck Z-scores over time, neither in men nor in women (table 3). At the lumbar spine, the intercept Z-score values were not significantly reduced in men or women. There was a small but significant increase in Z-scores at the lumbar spine over time in both groups (table 3 ). However, the mean estimated lumbar BMDs over the study period were not significantly different from the expected.

To examine changes in BMD between assessment points, individual patient Z-scores were compared in paired T-tests (figure 1). In the femoral neck, Z-scores for men decreased significantly from inclusion to the 5 -year follow-up visit (mean change in Z-score $-0.23,95 \%$ CI -0.43 to -0.03$)$, corresponding to a change in mean BMD of $-6.9 \%$ (95\% CI -4.5 to -9.3$)$ during the same period. After 5 years, no further reduction was seen (figure 1A). Lumbar spine Z-scores increased in both men and women over the study period (figure 1C,D), which was consistent with the results from the mixed linear effect models above.

\section{Predictors of BMD over time}

In the univariate analyses, higher body mass index (BMI) was the sole baseline factor that was associated with high Z-scores over time in both the femoral neck and lumbar spine in men as well as women (table 4). In men, none of the RA associated factors were significantly associated with the mean Z-scores over time. In women, higher age and postmenopausal status were associated with lower Z-scores in the femoral neck, and positive anti-CCP and a history of smoking predicted lower Z-scores in the lumbar spine (table 4). None of the RA associated factors were associated with consistent differences in change in BMD over time in univariate or multivariate analyses (see online supplementary files 1 and 2). 


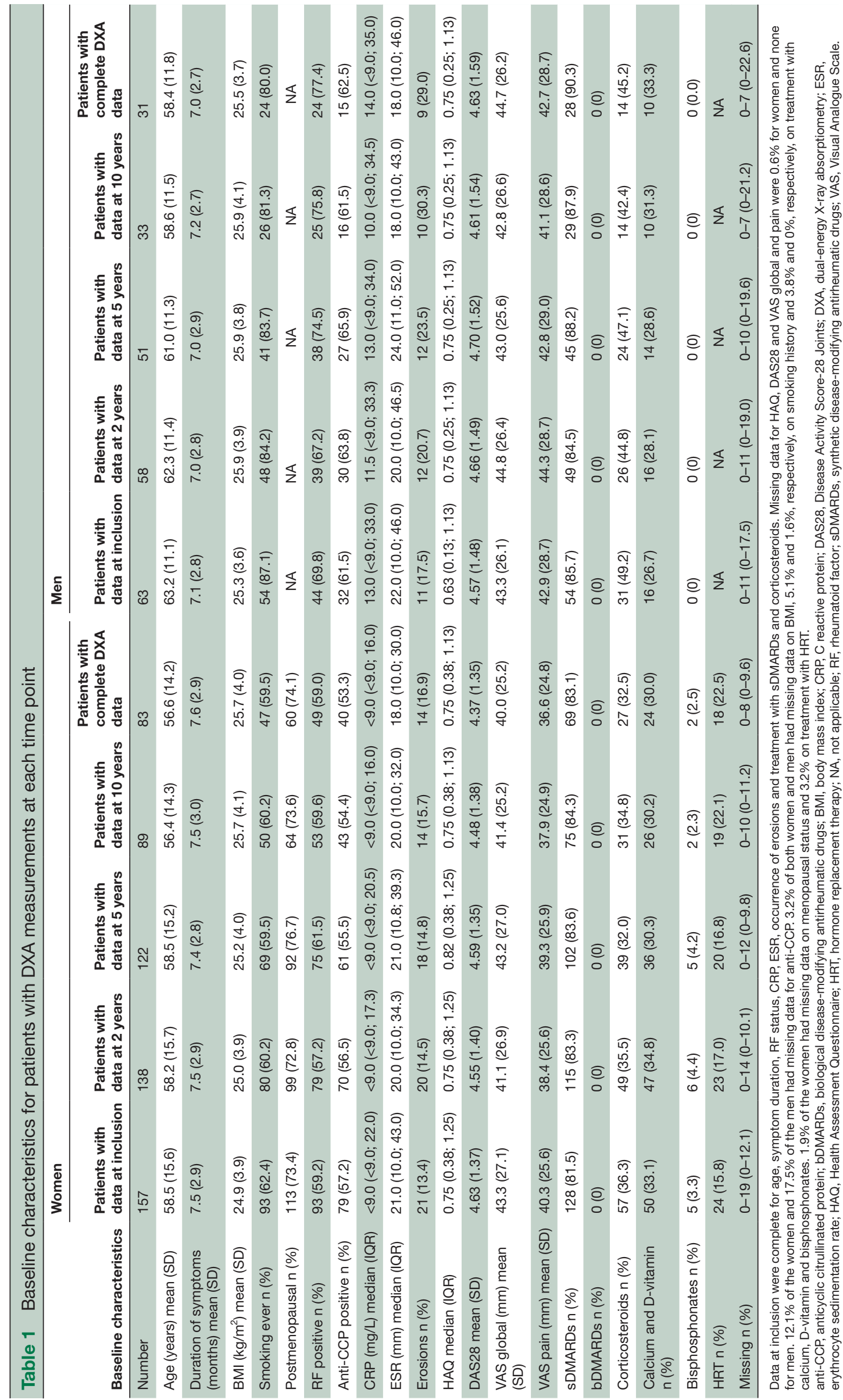




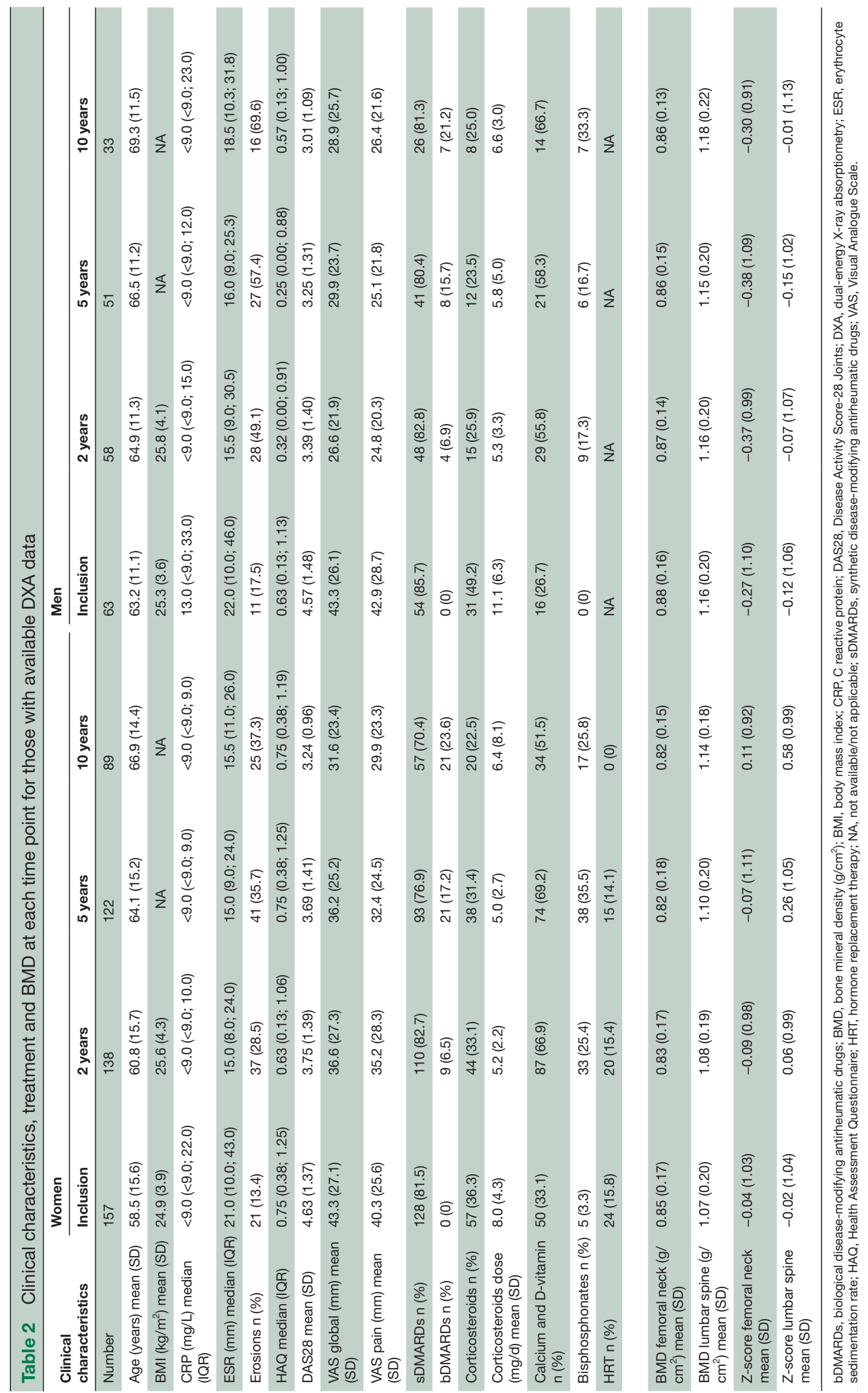


Table 3 Z-scores in the lumbar spine and the femoral neck over 10 years of time*

\begin{tabular}{|c|c|c|c|c|c|c|}
\hline & \multicolumn{2}{|c|}{ Mean Z-score } & \multicolumn{2}{|l|}{ Intercept } & \multicolumn{2}{|c|}{ Change/year } \\
\hline & Estimate & $95 \% \mathrm{Cl}$ & Estimate & $95 \% \mathrm{Cl}$ & Estimate & $95 \% \mathrm{Cl}$ \\
\hline Lumbar spine women & 0.057 & -0.100 to 0.213 & -0.043 & -0.205 to 0.119 & 0.039 & 0.025 to 0.053 \\
\hline Lumbar spine men & -0.053 & -0.294 to 0.187 & -0.094 & -0.365 to 0.176 & 0.023 & 0 to 0.045 \\
\hline Femoral neck women & -0.073 & -0.222 to 0.076 & -0.082 & -0.593 to 0.429 & 0.003 & -0.012 to 0.017 \\
\hline Femoral neck men & -0.327 & -0.570 to -0.085 & -0.352 & -0.614 to -0.090 & 0.004 & -0.014 to 0.023 \\
\hline
\end{tabular}

Bold text indicates significant associations.

*Mixed linear effect models.

Despite being a significant predictor of reduced BMD in women, age was not included in the multivariate analyses due to high correlation with menopausal status ( $\mathrm{r}$ $0.67, \mathrm{p}<0.001)$. In these models, BMI had a positive association with BMD Z-scores for men and women in both locations (table 5). In women, postmenopausal status (femoral neck only) and positive anti-CCP (lumbar spine only) were significantly associated with lower mean Z-scores over the 10-year period in the adjusted analyses (table 5).

Neither treatment with calcium and vitamin D, bisphosphonates or glucocorticosteroids at baseline had a significant impact on the mean Z-scores over 10 years in the femoral neck or the lumbar spine (table 4). Treatment with HRT at baseline predicted higher Z-scores over time in the lumbar spine in women in univariate (table 4) and multivariate analyses (table 5).

\section{DISCUSSION}

In this study of repeated BMD measurements in patients with recent onset of RA, men had reduced BMD in the femoral neck already at diagnosis, with significant but marginal further decline during the first 5 years of follow-up. This pattern was not seen in women, whose BMD in the femoral neck did not differ significantly from healthy women of the same age. The average cumulative decline of BMD in men during the first 5 years was $-6.9 \%$ (95\% CI -4.5 to -9.3$)$. Previous studies of repeated BMD measurements in men and women with early RA conducted in the early 1990's before the current practice of treat to target was implemented, have reported average annual rates of bone loss between $-0.28 \%$ and $-1.2 \%$ except for those with disease duration $<6$ months, ${ }^{8928}$ whereas studies conducted after the introduction of the biological DMARDs report annual rates between $-0.5 \%$ and $-1 \%$ per year. ${ }^{10} 29$
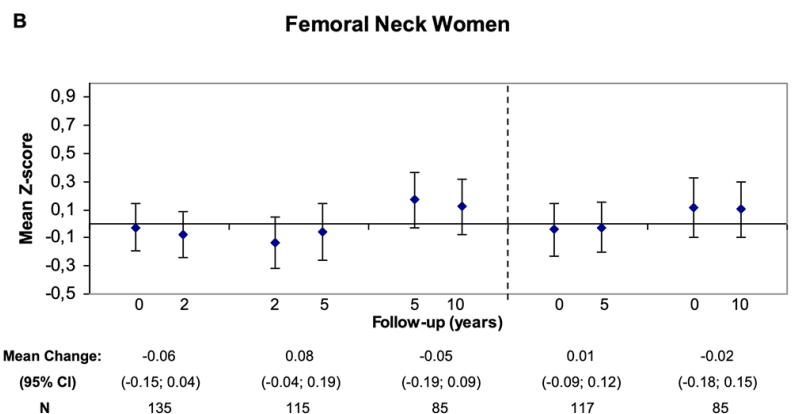

D

Lumbar Spine Women

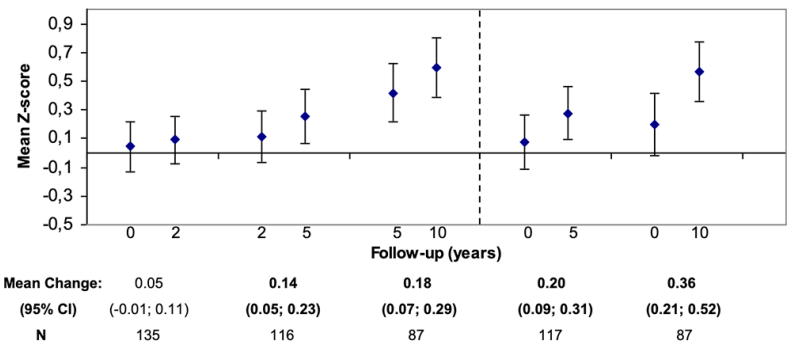

Figure 1 Pairwise comparisons of mean Z-scores between different follow-up visits, with mean changes of Z-scores. 


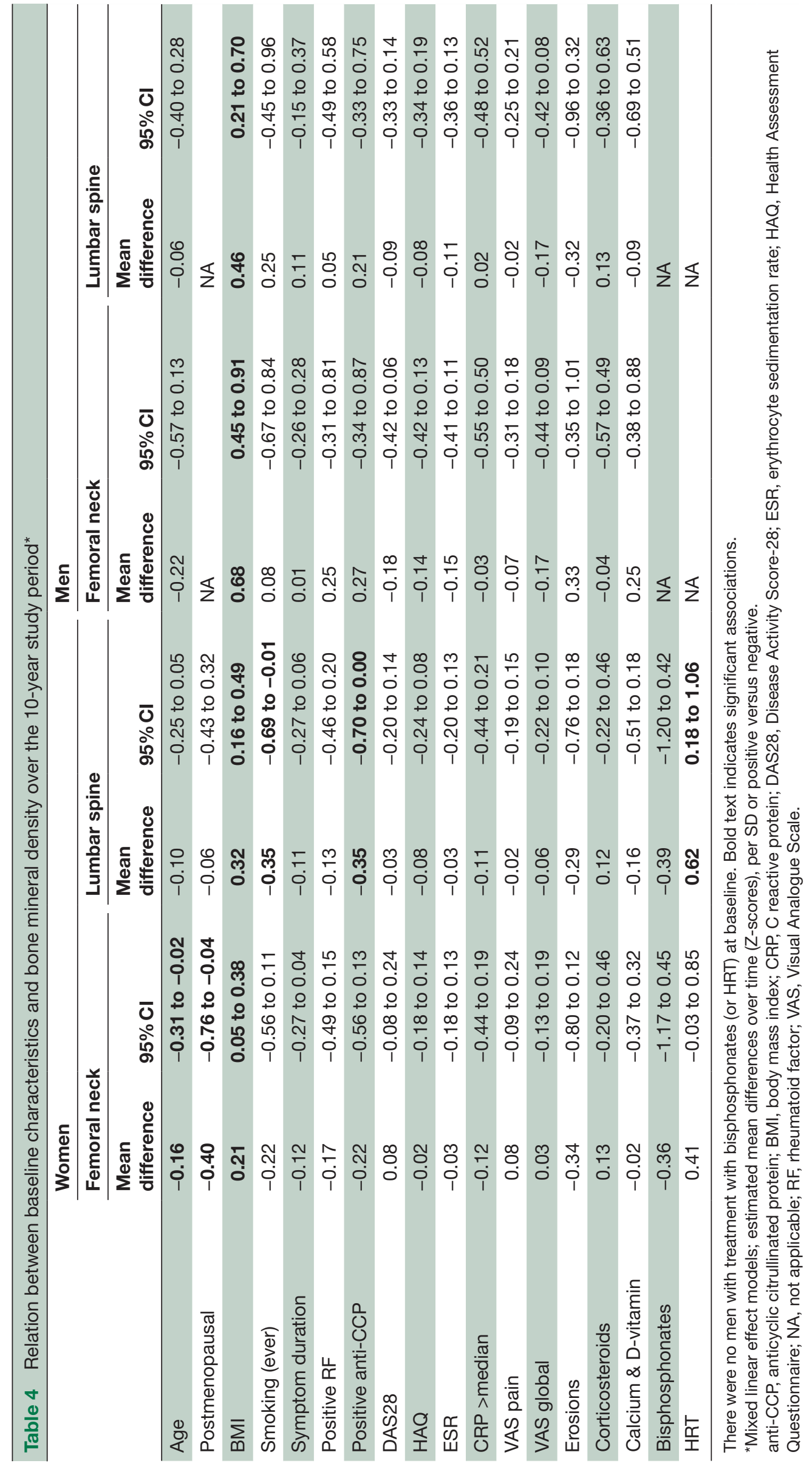

용

寻

흠 


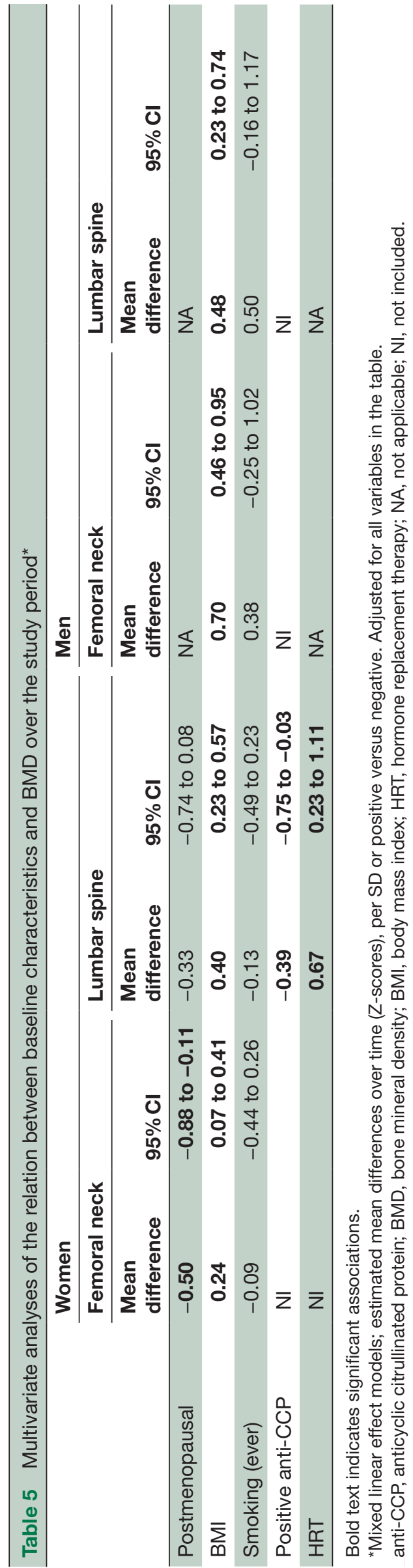

The early loss of bone mass found in men in this study is in line with previous studies where reduction in BMD has been most pronounced in the first years after RA diagnosis. ${ }^{8-10}$ Accelerated bone loss in men with RA has also been reported previously. ${ }^{7031}$ Potential explanations for the reduced BMD in men could include exposures that may predispose to both RA and low BMD in men, such as smoking ${ }^{32}$ and low androgen levels ${ }^{33}$ although the importance of low androgen levels for bone mass is debated, ${ }^{3435}$ and there are limited data on their impact in men with RA. ${ }^{4}$ Men also had more treatment with glucocorticosteroids, received antiosteoporotic treatment to a lesser extent and later and had more erosions at study start, although in this cohort none of these factors were significantly associated with Z-scores over time in neither the femoral neck nor lumbar spine.

Erosive disease has been presented as a risk factor for general osteoporosis in patients with $\mathrm{RA},{ }^{4-6}$ while associations with other markers of disease severity have been reported with inconsistent results. ${ }^{2369303136}$ In this study, none of the disease-related factors had a significant effect on BMD in men, whereas positive anti-CCP antibodies were associated with lower Z-scores in the lumbar spine in women, after adjustment for postmenopausal status, BMI and smoking. The inconsistent reports of associations with RA severity may be due to difficulties in obtaining a robust marker for cumulative disease activity and severity over time. The limited number of male patients at the 10-year follow-up, and the modest average change in Z-score over time, may contribute to the lack of significant associations of disease severity measures and BMD in this study. Furthermore, ongoing treatment with both antirheumatic and antiosteoporotic drugs and changes in therapy and disease course over time may limit long-term prediction of BMD. In this study, low BMI was the only risk factor that predicted low BMD at both locations in both men and women. In women, postmenopausal status was associated with lower Z-scores in the femoral neck after adjustment for BMI and smoking, whereas treatment with HRT at baseline was associated with higher Z-scores in the lumbar spine.

Although osteoporosis, spinal osteoporosis and vertebral fractures in particular, is a well-established side-effect of glucocorticosteroids, there are ongoing discussions on the topic of the potential positive effects on BMD due to the anti-inflammatory effects of glucocorticosteroids in RA. ${ }^{37}$ As with other disease related factors, there are conflicting results on the impact of glucocorticosteroids in patients with RA. ${ }^{13528} 37$ In this study, we did not find a significant association between baseline glucocorticosteroid treatment and BMD over time. The use of glucocorticosteroids was recorded at every follow-up visit but since treatment with Prednisone often varies over time, even in between follow-up visits, we decided against timedependent analyses in this study.

In the lumbar spine, Z-scores in both men and women increased over the study period. Divergence in bone loss between the hip and spine is often reported, and the main 
explanation for the higher BMD in the lumbar spine is the masking of bone loss by vertebral compression fractures, spinal degenerative changes and aortic calcifications. ${ }^{6} 103839$ In this study, spinal radiographs were not included and therefore we cannot evaluate such factors. Osteoarthritis in the lumbar spine is usually not considered to be a feature of RA, although we are not aware of any studies comparing osteoarthritis in the lumbar spine in patients with RA to healthy individuals. The increasing use of antiosteoporotic medication during the study period may also be part of the explanation for increasing Z-scores in the lumbar spine. Furthermore, the setting in which the study was performed, with regular DXA measurements, where antiosteoporotic treatment likely was changed based on DXA results, is favourable for preventing osteoporosis and may blunt or erase BMD changes during the course of the disease and limit longterm prediction of BMD. Such regular follow-up with DXA may likely contribute to a better BMD outcome in this study.

Limitations in this study are mainly due to the relatively small sample size and loss of patients for DXA follow-up measurements during the study period. Possibly, rapid bone loss during the last years before death could affect the estimated change in BMD in this study. Except for higher age, baseline characteristics of patients overall were not substantially different compared with the group with DXA data at all evaluations. Due to decreasing numbers with longer follow-up, power was limited for the predictor analyses, especially in men. A high prevalence of low D-vitamin levels has been observed in patients with RA, ${ }^{40}$ which is an aspect that may be relevant in this context. However, levels of D-vitamin were not available in this study. Furthermore, the results of this study, where patients were included between 1995 and 2005, may not apply to patients treated with bDMARDs in early disease. Further studies of such patients would be of interest. Finally, exposure during the period from symptom onset to inclusion in the study may influence BMD in this study.

Strengths in this study include the longitudinal design with a follow-up period of 10 years and the structured programme in which patients were examined. The fact that our patients were treated according to the general recommendations suggests that the results are highly representative to clinical practice. Overall, women with RA retained their BMD fairly well. In men with RA, however, early intervention with antiosteoporotic treatment could be beneficial and should be further studied.

\section{CONCLUSION}

This study indicates that femoral neck BMD is reduced at diagnosis of RA in men but not in women. The lower femoral neck BMD in men was sustained over the first 5 years with a statistically significant but marginal further decline. In the lumbar spine, BMD Z-scores increased over the observation period. Taken together, low bone mass in early RA may be a greater problem in men than in women.

\section{Author affiliations}

${ }^{1}$ Rheumatology, Department of Clinical Sciences, Malmö, Lund University, Malmö, Sweden

${ }^{2}$ Department of Rheumatology, Skåne University Hospital, Malmö and Lund, Sweden

${ }^{3}$ Rheumatology, Department of Clinical Sciences, Lund, Lund University, Lund, Sweden

${ }^{4}$ Clinical and Molecular Osteoporosis Research Unit, Department of Clinical Sciences, Lund University, Malmö, Sweden

${ }^{5}$ Department of Orthopedics, Skåne University Hospital, Malmö, Sweden

${ }^{6}$ Department of Rheumatology and Inflammation Research, Sahlgrenska Academy at Gothenburg University, Gothenburg, Sweden

Acknowledgements Christina Book, MD, PhD, initiated this project and performed a major part of the data collection. She passed away before the preparation of this manuscript. The authors would like to thank Lars Jehpsson for valuable advice on the statistical analyses.

Contributors LT participated in the study design and the data collection, performed the statistical analyses and wrote the first draft of the manuscript. MW managed the major part of the organisation of data for statistical analysis and participated in the analysis and interpretation of data. JÅN participated in the study design, the development of the patient questionnaires and the statistical analysis and in the analysis and interpretation of data. MK and KEÅ participated in the study design and in the analysis and interpretation of data. LJ participated in the study design, the design of the patient questionnaires and in the analysis and interpretation of data. CT conceived of the study, supervised the data management and the statistical analysis and helped draft the manuscript. All the authors helped in the revision of the manuscript and read and approved the final version.

Funding This work was supported by the Swedish Research Council (201502228), the Swedish Rheumatism Association (R-481821), Lund University (ALFSKANE-446501) and Region Skåne (REGSKANE-453511).

Competing interests None declared.

Patient consent for publication Not required.

Provenance and peer review Not commissioned; externally peer reviewed.

Data availability statement Data are available on reasonable request. The datasets generated and/or analysed during the current study are not publicly available due to European Union legislation (the General Data Protection Regulation), but a limited and fully anonymised dataset containing the individual patient data that support the main analyses is available from the corresponding author on reasonable request.

Open access This is an open access article distributed in accordance with the Creative Commons Attribution Non Commercial (CC BY-NC 4.0) license, which permits others to distribute, remix, adapt, build upon this work non-commercially, and license their derivative works on different terms, provided the original work is properly cited, appropriate credit is given, any changes made indicated, and the use is non-commercial. See: http://creativecommons.org/licenses/by-nc/4.0/.

ORCID iD

Lisa Theander http://orcid.org/0000-0002-3332-2108

\section{REFERENCES}

1 Hauser B, Riches PL, Wilson JF, et al. Prevalence and clinical prediction of osteoporosis in a contemporary cohort of patients with rheumatoid arthritis. Rheumatology 2014;53:1759-66.

2 Haugeberg G, Uhlig T, Falch JA, et al. Bone mineral density and frequency of osteoporosis in female patients with rheumatoid arthritis: results from 394 patients in the Oslo County rheumatoid arthritis register. Arthritis Rheum 2000;43:522-30.

3 Deodhar AA, Woolf AD. Bone mass measurement and bone metabolism in rheumatoid arthritis: a review. $\mathrm{Br} J$ Rheumatol 1996;35:309-22.

4 Tengstrand B, Hafström I. Bone mineral density in men with rheumatoid arthritis is associated with erosive disease and sulfasalazine treatment but not with sex hormones. J Rheumatol 2002;29:2299-305

5 Lodder MC, de Jong Z, Kostense PJ, et al. Bone mineral density in patients with rheumatoid arthritis: relation between disease severity and low bone mineral density. Ann Rheum Dis 2004;63:1576-80. 
6 Lodder MC, Haugeberg G, Lems WF, et al. Radiographic damage associated with low bone mineral density and vertebral deformities in rheumatoid arthritis: the Oslo-Truro-Amsterdam (OSTRA) collaborative study. Arthritis Rheum 2003:49:209-15.

7 Forslind K, Keller C, Svensson B, et al. Reduced bone mineral density in early rheumatoid arthritis is associated with radiological joint damage at baseline and after 2 years in women. $J$ Rheumatol 2003;30:2590-6.

8 Shenstone BD, Mahmoud A, Woodward R, et al. Longitudinal bone mineral density changes in early rheumatoid arthritis. $\mathrm{Br} J$ Rheumatol 1994;33:541-5.

9 Kroot EJ, Nieuwenhuizen MG, de Waal Malefijt MC, et al. Change in bone mineral density in patients with rheumatoid arthritis during the first decade of the disease. Arthritis Rheum 2001;44:1254-60.

10 Haugeberg G, Helgetveit KB, Førre Øystein, et al. Generalized bone loss in early rheumatoid arthritis patients followed for ten years in the biologic treatment era. BMC Musculoskelet Disord 2014;15:289.

11 Smolen JS, Landewé R, Bijlsma J, et al. EULAR recommendations for the management of rheumatoid arthritis with synthetic and biological disease-modifying antirheumatic drugs: 2016 update. Ann Rheum Dis 2017;76:960-77.

12 Alswat KA. Gender disparities in osteoporosis. J Clin Med Res 2017:9:382-7.

13 Dy CJ, Lamont LE, Ton QV, et al. Sex and gender considerations in male patients with osteoporosis. Clin Orthop Relat Res 2011;469:1906-12.

14 Genant HK, Cooper C, Poor G, et al. Interim report and recommendations of the World Health Organization task-force for osteoporosis. Osteoporos Int 1999;10:259-64.

15 Johnell O, Kanis JA, Oden A, et al. Predictive value of BMD for hip and other fractures. J Bone Miner Res 2005;20:1185-94.

16 Cummings SR, Marcus R, Palermo L, et al. Does estimating volumetric bone density of the femoral neck improve the prediction of hip fracture? A prospective study. Study of osteoporotic fractures Research Group. J Bone Miner Res 1994;9:1429-32.

17 Arnett FC, Edworthy SM, Bloch DA, et al. The American rheumatism association 1987 revised criteria for the classification of rheumatoid arthritis. Arthritis Rheum 1988;31:315-24.

18 Smolen JS, Aletaha D, Bijlsma JWJ, et al. Treating rheumatoid arthritis to target: recommendations of an international task force. Ann Rheum Dis 2010;69:631-7.

19 Rydell E, Forslind K, Nilsson Jan-Åke, et al. Smoking, body mass index, disease activity, and the risk of rapid radiographic progression in patients with early rheumatoid arthritis. Arthritis Res Ther 2018;20:82.

20 Rydholm M, Book C, Wikström I, et al. Course of grip force impairment in patients with early rheumatoid arthritis over the first five years after diagnosis. Arthritis Care Res 2018;70:491-8.

21 Ekdahl C, Eberhardt K, Andersson SI, et al. Assessing disability in patients with rheumatoid arthritis. Use of a Swedish version of the Stanford health assessment questionnaire. Scand J Rheumato 1988;17:263-71.

22 Geborek P, Nitelius E, Noltorp S, et al. Population based studies of biological antirheumatic drug use in southern Sweden: comparison with pharmaceutical sales. Ann Rheum Dis 2005;64:1805-7.

23 Eriksson JK, Askling J, Arkema EV. The Swedish rheumatology quality register: optimisation of rheumatic disease assessments using register-enriched data. Clin Exp Rheumatol 2014;32:S-147-9.
24 Callréus M, McGuigan F, Ringsberg K, et al. Self-reported recreational exercise combining regularity and impact is necessary to maximize bone mineral density in young adult women: a population-based study of 1,061 women 25 years of age. Osteoporos Int 2012;23:2517-26.

25 Lenora J, Ivaska KK, Obrant KJ, et al. Prediction of bone loss using biochemical markers of bone turnover. Osteoporos Int 2007;18:1297-305.

26 Karlsson MK, Gärdsell P, Johnell O, et al. Bone mineral normative data in Malmö, Sweden. Comparison with reference data and hip fracture incidence in other ethnic groups. Acta Orthop Scand 1993:64:168-72.

27 Alwis G, Linden C, Stenevi-Lundgren S, et al. A school-curriculumbased exercise intervention program for two years in pre-pubertal girls does not influence hip structure. Dyn Med 2008;7:8.

28 Gough AK, Lilley J, Eyre S, et al. Generalised bone loss in patients with early rheumatoid arthritis. Lancet 1994;344:23-7.

29 Güler-Yüksel M, Allaart CF, Goekoop-Ruiterman YPM, et al. Changes in hand and generalised bone mineral density in patients with recent-onset rheumatoid arthritis. Ann Rheum Dis 2009;68:330-6.

30 Güler-Yüksel M, Bijsterbosch J, Goekoop-Ruiterman YPM, et al. Bone mineral density in patients with recently diagnosed, active rheumatoid arthritis. Ann Rheum Dis 2007;66:1508-12.

31 Keller C, Hafström I, Svensson B, et al. Bone mineral density in women and men with early rheumatoid arthritis. Scand $J$ Rheumatol 2001;30:213-20.

32 Bergström U, Jacobsson LTH, Nilsson Jan-Åke, et al. Pulmonary dysfunction, smoking, socioeconomic status and the risk of developing rheumatoid arthritis. Rheumatology 2011;50:2005-13.

33 Pikwer M, Giwercman A, Bergström U, et al. Association between testosterone levels and risk of future rheumatoid arthritis in men: a population-based case-control study. Ann Rheum Dis 2014;73:573-9.

34 Mohamad N-V, Soelaiman I-N, Chin K-Y. A concise review of testosterone and bone health. Clin Interv Aging 2016;11:1317-24.

35 Hoppé E, Morel G, Biver E, et al. Male osteoporosis: do sex steroids really benefit bone health in men? Joint Bone Spine 2011;78:S191-6.

36 Book C, Karlsson M, Akesson K, et al. Disease activity and disability but probably not glucocorticoid treatment predicts loss in bone mineral density in women with early rheumatoid arthritis. Scand $J$ Rheumatol 2008;37:248-54.

37 van der Goes MC, Jacobs JWG, Jurgens MS, et al. Are changes in bone mineral density different between groups of early rheumatoid arthritis patients treated according to a tight control strategy with or without prednisone if osteoporosis prophylaxis is applied? Osteoporos Int 2013;24:1429-36.

38 Lenora J, Akesson K, Gerdhem P. Effect of precision on longitudinal follow-up of bone mineral density measurements in elderly women and men. J Clin Densitom 2010;13:407-12.

39 Tenne M, McGuigan F, Besjakov J, et al. Degenerative changes at the lumbar spine--implications for bone mineral density measurement in elderly women. Osteoporos Int 2013;24:1419-28.

40 Lee $\mathrm{YH}, \mathrm{Bae} \mathrm{S}-\mathrm{C}$. Vitamin D level in rheumatoid arthritis and its correlation with the disease activity: a meta-analysis. Clin Exp Rheumatol 2016;34:827-33. 